\title{
A Brief Exploration into Scientific Thought and Aesthetic Ideas of Plato
}

\author{
Zhide Wang \\ HuaiHai Institute of Technology, College of Humanities, Jiangsu, Lianyungang 222005
}

\begin{abstract}
In the long history of the development of western aesthetics, the relationships between science and aesthetics, especially the relationship between mathematics and aesthetics, are very close. Ancient Greek culture has a notable feature. It has a keen interest that can be hardly compared with by any other ethnic groups on the pursuit of nature. Because they are dissatisfied with the imagination in the poetic world of myth and they are trying to rationally explain the order of nature, its evolution and movement. The natural philosophers of ancient Greece study science and the aesthetic issues at the same time. As the beginner of the western culture, Plato is in behalf of the ancient Greece representatives. He is more than a philosopher. He is a very important influential scientist whose scientific ideas are closely related to the aesthetic concept.
\end{abstract}

Keywords- Plato, "Platonic solid" slight exploration into aesthetics

\section{THE INTERNAL CONNECTION OF BEAUTY AND} SCIENCE

In the long history of the development of western aesthetics, the relationships between science and aesthetics, especially the relationship between mathematics and aesthetics, are very close. In every history of philosophy for students, the first thing mentioned is that philosophy began with Thales, who said that everything is made of water. There is ample reason to feel respect for Thales, though perhaps rather as a man of science than as a philosopher in the modern sense of the world. Thales was a native of Miletus, in Asia Minor, a flourishing commercial city. Thales is said to have travelled in Egypt, and to have thence brought to the Greeks the science of geometry. He seems to have discovered how to calculate the distance of a ship at sea from observations taken at two points on land, and how to estimate the height of a pyramid from the length of its shadow. "Thales is the first person in history that has come to Egypt and brought back the professional knowledge of geometry to the Greeks. He has found a lot of subjects on his own and taught many other basic principles to his inheritors." "1] Mathematics, in the sense of demonstrative deductive argument, begins with Pythagoras. The profound influence of mathematics on philosophy, partly owing to him, has ever since his time. Pythagoras and his school have improved the abstract concept of mathematics to an outstanding status and taken mathematics as the foundation stone of the world. The combination of mathematics and aesthetics began with Pythagoras. It is also the Pythagoreans opened a great prelude to the development of scientific and aesthetic thought in ancient Greece. They have opened the world of beauty and mathematics and the unified landscape with science subjects shouldering side by side. This kind of default on the unified image is developed through Democritus. When it comes to the age of Plato and Aristotle, it has become an aesthetic concern of the natural philosophers in the process of scientific inquiry.

Ancient Greek culture has a notable feature. It has a keen interest that can be hardly compared with by any other ethnic groups on the pursuit of nature. Because they are dissatisfied with the imagination in the poetic world of myth and they are trying to rationally explain the order of nature, its evolution and movement. There are some famous schools, such as Miletus from Ionia, Pythagoras and his followers, the Eleatic school and the atomic theory school and so on. Among them, the sages from Ionia are the pioneers of the spirit of rationalism. Representatives are Thales, Anaximander, Heraclitus, and Anaxagoras and so on. According to Thales, he thought that water is the original substance, out of which all others are formed; and he maintained that the earth rests on water. Russell has once commented that, "the importance of Thales is that he has bluntly raised these issues and his reply does not concern things about myth." As for Anaximander who has raised thoughts on the universe, he believes that "There was an eternal motion, in the course of which was brought about the origin of the worlds." "The worlds were not created, as in Jewish or Christian theology, but evolved." [2] Pythagoras was intellectually one of the most significant men that ever lived in the world. Since his time, mathematics, in the sense of demonstrative deductive argument has profound influence. Mathematics thus appeared as an independent subject in Greece.

\section{THE SCIENTIFIC SUPPORT OF PLATO'S THEORY OF IDEAS}

Plato was born in 427 B.C. and died in 347 B.C. His parents were well-do-do aristocrats. At the beginning he studied from other teachers, learning poetry, music, painting and philosophy. At the age of twenty-eight, he became a pupil of Socrates, for whom he had a profound affection and respect.

Plato is the most important thinkers of ancient Greece. He attached particular importance to the concept of mathematics for his theory of ideas. In Plato's academy, the study of natural science and mathematics are positive. They are so important that they have even become tickets to enter the academy. Those who do not understand geometry will not be admitted. Thales, a famous mathematician who has found the three-dimensional geometry at that period of time, proved that there were no more than five regular polyhedrons. Menaechmus is a mathematician and geometer who was known for his apparent discovery of conic sections. His teacher Eudoxus was at the Platonic Academy. Through their 
research, Platonic Academy has sorted out the deductive method of mathematics and geometry, which has laid a foundation for the advent of the world's first a mathematical writings-- Euclid's Elements.

When Plato was fundamental questioning the world, he brought out his "theory of ideas", believing that theory is the essence of beauty and that the essence of beauty does not lie in the specific matters. However, when he was trying to explain the relationship between the beauty of theory and the beauty of things, Plato found it quite difficult. In his view, the number not only constitutes things in the world, but it is also the only way to achieve the theory. In one of his articles "Philebus", Plato has provided us with the shapes relevant to the numbers and the singular numbers and the whole numbers and he maintained that they were absolutely beautiful. "The beauty of form, as what I have said, does not refer to the beauty of the animals or paintings, about which a majority of people have already known. Instead, the beauty just lies in the plane-shaped and cubic that are formed with a straight line and a circle as well as the straight line and the circle formed with the foot, regulations, and the moment...The beauty of these shapes are unlike the other things whose beauties are relative. They always have the absolute beauty according to their essence". He also has some views about music, "my meanings are that some voices are soft and clear, resulting in an integrated and pure tone. As for these voices, their beauty is not relative. Their beauty does not come from the relationship with other things. Their beauty comes from their essence, which is quite absolute." [4]

\section{III. “PlatONIC SOLID” IN SCIENTIFIC DISCOVERY}

Plato believes that knowledge of the truth shall be recognized through the geometry. The influence of physics and the experience in the world should be set aside. In his view, the beauty of geometry is not only the beauty of form, but also the substantial construction of the real world. Plato's view of the universe has been criticized by many people. Although it is the case, what provides much food for thought is that many scientific researches and scientific discoveries are found to be related with the "platonic solid".

"In ancient Greece, Pythagoras was the first one to apply the harmonious thoughts of numbers to the research of the structure of the universe, and thus the development of mathematical astronomy began." [6] At that time, "every Greek feels that objects should reflect the beauty of mathematics" [7] Plato has inherited and developed the Pythagorean Theory of Harmony in the universe in the Greek cultural patterns at that time. In Euclidean geometry, a Platonic solid is a regular, convex polyhedron. The faces are congruent, regular polygons, with the same number of faces meeting at each vertex. There are five Platonic solids; their names are derived from their numbers of faces. The aesthetic beauty and symmetry of the Platonic solids have made them a favourite subject of geometers for thousands of years. They are named for the ancient Greek philosopher Plato, who theorized that the classical elements were constructed from the regular solids. As for Plato, the shape and size of the various components of the universe are in line with a certain proportion, saying that "the best combination of things is that it combines with the most complete blend, but to achieve this goal , the ratio is extremely important." ${ }^{[8]}$ Plato pays special attention to the five regular Platonic solids. At that period of time, the Greeks believed that all things are composed of the following four elements: fire, water, soil and air. Plato believed that the shapes of these four elements are tetrahedron, cube or hexahedron, octahedron and dodecahedron. Their different shapes make their nature different. As for dodecahedron, there are no correspondent basic elements, and later Aristotle believed that it is the "ether".

The mathematical world view of Plato is taken as an astronomy cognitive paradigm. The mathematical world view of Plato has a direct impact on the development of modern western astronomy. He was convinced that "God created the world in accordance with the principle of the perfect number, so the fundamental mathematical harmony, that is to say, the so-called celestial music, is the reason that can be found for the real motions of the planets." [9] Jiang Qian is a domestic scholar. In his article "Research on the scientific knowledge and the aesthetic function of 'Platonic solid", he points out that Johannes Kepler has designed the structure of the solar system with Platonic solid carefully. For the largest of Saturn orbit celestial sphere, the inner surface of the mosaic (access) is a cube, whose size just makes Jupiter's orbit celestial sphere inscribe in the midpoint of its surface. There is a regular tetrahedron inscribed in the Jupiter, with its inner side of Mars Celestial. Then it is the dodecahedron, with its inner side of the celestial sphere of the Earth's orbit. Next it is the icosahedrons, within Venus orbit the celestial sphere inscribed within a regular octahedron, the orbit of Mercury celestial cut it. This is the result, although it has some distance from the observed values. Kepler thinks that this error is caused by the fact that an ellipse is a particular class of mathematical shapes that resemble a stretched out circle. After a long gestation, Kepler completed his masterpiece "The Harmony of the Worlds" in 1618.

In the past we studied a lot of the forms of mechanical movement of the materials. However, we made relatively less researches on the forms of harmonious overall movement of substances. As for this kind of universal and harmonious mathematical expressions, Engels has once said that due to the fact that the existence of natural science does not last for a long time, there are many defects on this aspect according to our understandings. If we know the movement forms of the materials, we would know the material itself. The development of the modern science increasingly reveals the movement form of the material world and its inherent relationship with the subject of mathematics. They have revealed "Platonic solid" and the implication of the golden section in the harmonious movement of substances universality from a variety of perspectives, such as the movement of celestial objects from the macro perspective, the growth morphology of organisms from the medium perspective, or the material physical and chemical structure from the micro perspective. The Scientist Friedrich Cramer has pointed out in his book "Chaos and Order" that golden number reveals a common and harmonious element in the 
chaotic and orderly boundary. Under the guidance of scientific and holistic thinking, he relates the nonlinear science and chaos theory with the geometry by Pythagoras, Plato and Kepler. In this way, he holds the view that it is the discovery of these laws that embody the amazing force of the intuition "harmonization". Just like numerology, some of the things that have long been considered by Kepler in his early work "The Sacred Mystery of the Cosmos" have again unwittingly entered into the description of the physical system, but this time it is coming in from the back door.

Scientific discoveries cannot be separated from the creation of mathematical concepts. The golden section method is one of the most important structural tools. Plato believed that the truth of mathematics had nothing to do with the perception. The truth of mathematics involves only symbols. In terms of numbers, speaking in the strict sense, it is the existence form of the world. "The numbers are, in a certain precise sense, formal. The facts which verify various propositions asserting that various collections each have two members, have in common, not a constituent, but a form. In this they differ from propositions about the Statue of Liberty, or the moon, or George Washington." [10] Einstein once pointed out that we were able to use the structure of pure mathematics to discover the concepts and the laws that link these concepts, and that these concepts and the laws are the keys to understanding natural phenomena.

\section{REFERENCES}

[1] MO De, "The original study of the Elements by Euclid", Mongolia People's Publishing House, Edition 1992, Page 15

[2] BERTRAND RUSSELL: 《A History of Western Philosophy》, The Commercial Press, 1963 Edition, Page 53、Page 144、Page 176, Page 175, Page 205

[3] BERTRAND RUSSELL: 《A History of Western Philosophy》, The Commercial Press, 1963 Edition, Page 53、Page 144、Page 176, Page 175, Page 205

[4] ZHU Guangqian : 《Plato's literary dialogue set》, People's Literature Publishing House, Edition 1980, Page 298.

[5] BERTRAND RUSSELL: 《A History of Western Philosophy》, The Commercial Press, 1963 Edition, Page 53、Page 144、Page 176, Page 175, Page 205

[6] LUO Ban : 《The origins of Greek thought and the scientific spirit $\rangle$, The Commercial Press, 1965 Edition, Page 80.

[7] BERTRAND RUSSELL: 《A History of Western Philosophy》, The Commercial Press, 1963 Edition, Page 53、Page 144、Page 176, Page 175, Page 205

[8] Plato : 《Plato's Complete Works》 (Volume Three), People's Publishing House, Edition 2003, Page 282

[9] Dampier.W.C. : 《The History of Science》, The Commercial Press, 1975 Edition, Page 193.

[10] BERTRAND RUSSELL: 《A History of Western Philosophy》, The Commercial Press, 1963 Edition, Page 53、Page 144、Page 176, Page 175, Page 205. 